\title{
Presión en la reparación de manguito rotador con suturas transóseas y Mason-Allen modificado
}

\section{Pressure on the Rotator Cuff Repair with Transosseous Sutures and Modified Mason-Allen}

\author{
Julio José Contreras Fernández ${ }^{1}$ Oscar Sepúlveda Osses ${ }^{2} \quad$ Nicolás Prado Esper $^{2} \quad$ Ricardo Guzmán Silva $^{1}$ \\ Rodrigo Liendo Verdugo ${ }^{3}$ Francisco Soza Rex ${ }^{3}$
}

\footnotetext{
${ }^{1}$ Instituto Traumatológico, Santiago, Región Metropolitana, Chile

${ }^{2}$ Hospital del Trabajador, Santiago, Región Metropolitana, Chile

3 Pontificia Universidad Católica de Chile, Santiago, Región

Metropolitana, Chile
}

Address for correspondence Julio José Contreras Fernández, Instituto Traumatológico, San Martín 771, Santiago, Región Metropolitana, Chile (e-mail: Juliocontrerasmd@gmail.com).

Rev Chil Ortop Traumatol 2021;62:19-26.

\section{Resumen \\ Palabras Clave \\ - manguito de los rotadores \\ - presión \\ - sutura \\ - técnicas de sutura \\ - traumatismos de los tendones \\ - tendones}

\section{Abstract}

Objetivo Comparar el promedio de curva de presión de contacto y el porcentaje de presión de contacto residual final en la interfase tendón-huella de una reparación transósea (TO) realizada con nudos cruzados y una configuración Mason-Allen modificada (MAM).

Métodos Se utilizaron ocho hombros de cordero para simular una rotura de manguito rotador. Se midió la presión con un sensor digital. Se registró la presión basal durante la aplicación de carga cíclica y al final de la intervención. Se compararon dos reparaciones: dos túneles TOs con nudos cruzados (TOCs) $(n=4)$ y dos puntos MAMs $(n=4)$ utilizando suturas MaxBraid \#2 (Zimmer Biomet, Warsaw, IN, EEUU). Se realizaron 1.000 ciclos, con una frecuencia de $2 \mathrm{~Hz}$ y una carga de $30 \mathrm{~N}$. Se utilizó el test de $t$ de Student, y se consideraron significativos valores de $p<0,05$.

Resultados El promedio de curva de presión de contacto en las piezas que fueron reparadas con suturas TOCs fue de $86,01 \pm 8,43 \%$, mientras que con MAM fue de $73,28 \pm 12,01 \%(p<0,0004)$. El promedio del porcentaje residual al final del ciclado fue de $71,57 \%$ para suturas TOCs y de $51,19 \%$ para MAM $(p<0,05)$.

Conclusión La reparación TOC presenta mayor promedio de curva de presión de contacto y mayor porcentaje de presión de contacto residual final en la interfase tendón-huella que la reparación con sutura MAM luego de carga cíclica estandarizada, lo que podría traducirse en una mejor cicatrización del tendón.

Nivel de Evidencia Estudio de ciencia básica.

Objective To compare the average contact pressure curve and the percentage of final residual contact pressure at the tendon-footprint interphase of a transosseous (TO) received

June 28,2020

accepted

January 21, 2021
DOI https://doi.org/

$10.1055 / \mathrm{s}-0041-1728736$ ISSN 0716-4548. (c) 2021. Sociedad Chilena de Ortopedia y Traumatologia. All rights reserved.

This is an open access article published by Thieme under the terms of the Creative Commons Attribution-NonDerivative-NonCommercial-License, permitting copying and reproduction so long as the original work is given appropriate credit. Contents may not be used for commercial purposes, or adapted, remixed, transformed or built upon. (https://creativecommons.org/ licenses/by-nc-nd/4.0/)

Thieme Revinter Publicações Ltda., Rua do Matoso 170, Rio de Janeiro, RJ, CEP 20270-135, Brazil 


\author{
Keywords \\ - rotator cuff \\ - pressure \\ - suture \\ - suture techniques \\ - tendon injuries \\ - tendons
}

repair performed with crossover sutures or a modified Mason-Allen (MMA) configuration.

Methods Eight lamb shoulders were used to simulate a rotator cuff tear. The pressure was measured with a digital sensor. The baseline pressure was recorded during the application of the cyclic load and at the end of the intervention. Two repairs were compared: 2 crossover TO (CTO) sutures $(n=4)$ and 2 MMA sutures MMA $(n=4)$, using MaxBraid \#2 (Zimmer Biomet, Warsaw, IN, US) sutures. A thousand cycles were performed, with a frequency of $2 \mathrm{~Hz}$ and a $30-\mathrm{N}$ load. The Student $t$-test was used, and significance was set at $p<0.05$.

Results The average contact pressure curve was of $86.01 \pm 8.43 \%$ for parts repaired with CTO sutures, and of $73.28 \pm 12.01 \%$ for those repaired with MMA sutures $(p<0.0004)$. The mean residual percentage at the end of cycling was of $71.57 \%$ for CTO sutures, and of $51.19 \%$ for MMA sutures $(p<0.05)$.

Conclusion The CTO repair shows a higher average contact pressure curve and a higher percentage of final residual contact pressure at the tendon-footprint interphase than the MMA suture repair after standardized cyclic loading, potentially resulting in improved tendon healing.

Level of Evidence Basic Science Study.

\section{Introducción}

La reparación artroscópica del manguito rotador ha presentado un aumento constante en el último tiempo. ${ }^{1}$ Los resultados clínicos y funcionales son de buenos a excelentes en el corto y largo plazos en la mayoría de los casos ${ }^{2-5}$ sin embargo, las tasas de rerotura siguen siendo considerables, descritas entre un $11 \%$ y un $68 \%$ en algunas series, incluso alcanzando un $94 \%$ en algunos estudios. ${ }^{6-8}$

La cirugía de reparación del manguito rotador busca establecer una interfase fibrovascular entre el tendón y la huella, que es necesaria para la curación y restauración de la inserción fibrocartilaginosa (entesis); para lograr esto, se busca un constructo que maximice el contacto presurizado entre el tendón y el hueso mientras mantiene la resistencia mecánica contra la carga fisiológica. ${ }^{9}$ La rerrotura se asocia a factores del paciente y de la reparación (factores anatómicos). Respecto a los factores del paciente, se asocian a aumento de la edad, mayor tamaño de la rotura (compromiso de múltiples tendones), menor calidad tendínea, atrofia muscular, degeneración grasa (Goutallier $\geq$ a 3), retracción tendínea, mayor tiempo de evolución, y presencia de comorbilidades (tabaquismo, diabetes, hipercolesterolemia, alcoholismo, obesidad e hipertensión). ${ }^{7,10}$ Respecto a los factores anatómicos de la reparación, depende de la tensión del constructo, perfusión tisular, el micromovimiento en la interfase tendón-huella, y la presión y área de contacto en la huella. ${ }^{11}$ El principio subyacente es que una mayor magnitud y distribución del área de contacto del tendón al hueso dará como resultado una mayor posibilidad de curación del tendón. ${ }^{12}$

Diversos estudios biomecánicos ${ }^{5,13}$ de la reparación en doble fila (DF) han demostrado un aumento de la resistencia a la falla por carga, áreas de contacto y presiones mejoradas, y una disminución de la formación de espacios (gap) en la interfase tendón-huella en comparación con la reparación en fila única (FU). Sin embargo, las anclas proporcionan una baja resistencia, tienden a aflojarse en el hueso osteoporótico, dejan de tener un contacto óptimo a nivel de la huella del tendón supraespinoso, producen osteólisis de la tuberosidad mayor, son de revisión difícil, y generan aumento del costo. $^{14,15}$ Los sitios de falla pueden incluir el tendón, la sutura, el hueso, o el ancla, así como las interfases entre el hueso y el ancla, el ancla y la sutura, o entre la sutura y el tendón. ${ }^{16}$ Esto ha llevado a la creación de nuevos tipos de anclas, como las anclas de sutura. ${ }^{17,18}$

La técnica transósea (TO) permite maximizar el área de contacto de la interfase tendón-huella ${ }^{19}$ y reducir el movimiento en la interfase tendón-hueso. ${ }^{20}$ Además de este aspecto mecánico, la técnica TO permite preparar con precisión el lado óseo de la lesión sin ningún riesgo o complicación, como la extracción del ancla y/o una osteólisis de la tuberosidad mayor. ${ }^{21,22}$

El uso de técnicas de sutura transósea es eficiente y reproducible en la reparación artroscópica de roturas del manguito rotador. ${ }^{23}$ Además, existe un potencial mayor de cicatrización por el contacto directo del tendón con el hueso (sin material de anclas interpuesto) y con células madres mesenquimales provenientes de los túneles óseos del humero proximal. ${ }^{24-26}$

Finalmente, el año 2009, Burkhart et al. ${ }^{27}$ describieron un mecanismo por el cual el aumento de la tensión aplicado al constructo genera una resistencia creciente a la falla estructural al generar un aumento progresivo de las fuerzas de compresión en la huella del tendón, llamado autorreforzamiento (self-reinforcement). Las fuerzas de compresión creadas en la huella aumentan la resistencia a la fricción entre el tendón y el hueso, reduciendo así la formación de espacios entre las dos superficies. 
La reparación en FU con técnica Mason-Allen modificada (MAM) según Habermayer ${ }^{28-30}$ ofrece una reparación fácil de realizar, y proporciona una excelente resistencia de fijación inicial, que permite una integración osteofibroblástica duradera del manguito reinsertado; posee buenos a excelentes resultados clínicos reproducibles, con una tasa de rerotura del $25 \%$, que es comparable con los resultados de la reparación abierta. ${ }^{30}$

El objetivo de este estudio es comparar el promedio de curva de presión de contacto y el porcentaje de presión de contacto residual final en la interfase tendón-huella de una reparación TO realizada con nudos cruzados (TOC) y una configuración MAM. Nuestra hipótesis es que la configuración TOC tendrá un mayor promedio de curva de presión de contacto y mayor porcentaje de presión de contacto residual final en la interfase tendón-huella.

\section{Materiales y métodos}

\section{Modelo animal}

Ocho hombros frescos congelados de cordero (Ovis orientalis aries) de 6 meses de edad obtenidos de una empresa frigorífica local (corte "paleta oyster", Frigorífico Simunovic Ltda., Punta Arenas, Región de Magallanes y Antártica Chilena, Chile) fueron descongeladas a temperatura ambiente la noche previa a las pruebas biomecánicas (18 horas en total, con piezas anatómicas a temperatura ambiente totalmente descongeladas). Se seleccionó el tendón infraespinoso por poseer características anatómicas y funcionales equivalentes a las del tendón supraespinoso de humanos. ${ }^{31}$ Los especímenes fueron disecados de forma estandarizada, eliminando todo el tejido blando en relación a la diáfisis humeral, las fosas subescapular y supraespinosa de la escápula, con el objetivo de aislar el músculo infraespinoso y su tendón. No se evidenciaron alteraciones del manguito rotador en ninguno de los espécimenes. Luego, se realizó una disección cuidadosa del tendón infraespinoso, que fue suturado a una cinta de nailon polipropileno con una sutura tipo Krackow con MaxBraid \#2 (Zimmer Biomet, Warsaw, IN, EEUU), con tal de poder manipular el músculo y el tendón sin desgarrarlos; la cinta fue sujetada mediante una abrazadera a un actuador lineal, con una celda de carga intermedia (-Figura 1). Las piezas fueron irrigadas intermitentemente con solución salina $(\mathrm{NaCl} 0,9 \%)$ a lo largo de cada ensayo para prevenir la deshidratación de la muestra.

Se utilizó un sistema a medida para generar tensiones cíclicas a nivel del tendón infraespinoso (-Figura 2). El modelo constó de tres partes fundamentales: un soporte modular de altura regulable, un soporte ajustable para guiar el sistema de suturas, y un actuador lineal, con una celda de carga intermedia. El actuador fue programado para ciclar a una frecuencia de $2 \mathrm{~Hz}$, con una carga de $30 \mathrm{~N}$, realizando un total de 1.000 ciclos. Se registró la presión de contacto cada 50 ciclos. La diáfisis humeral fue fijada en una abrazadera metálica. Luego, se ajustó el soporte modular para asegurar el paralelismo del tendón con la vertical (se utilizó un nivel), generando un vector de tracción del tendón en $0^{\circ}$ de abducción y $0^{\circ}$ de rotación (-Figura 3 ).

\section{Rotura del manguito rotador}

En cada cabeza humeral, se identifica la orientación de la tuberosidad mayor, y es demarcada con una aguja de Kirschner de $1,5 \mathrm{~mm}$. Seguidamente, se identifica la punta de la tuberosidad y se realiza una rotura de espesor total y ancho completo con un bisturí $\mathrm{n}^{\circ} 15$, liberando toda la inserción del tendón en la huella, para luego aplanarla con una raspa, con tal de facilitar la instalación de sensores de presión (-Figura 4).

\section{Medición de presión en la interfase tendón-huella}

Para medir la presión en la interfase tendón-huella, se utilizó un sensor de presión digital Tekscan FlexiForce (Tekscan, Inc., Boston, MA, EEUU), el cual se posicionó entre el tendón y la huella, quedando fijado gracias a la reparación realizada, y cubriendo el área total de la huella; este sensor registra los cambios de presión en el tiempo, y los almacena en un computador para su análisis posterior. Se registró la presión basal al inicio del experimento (tiempo 0), durante

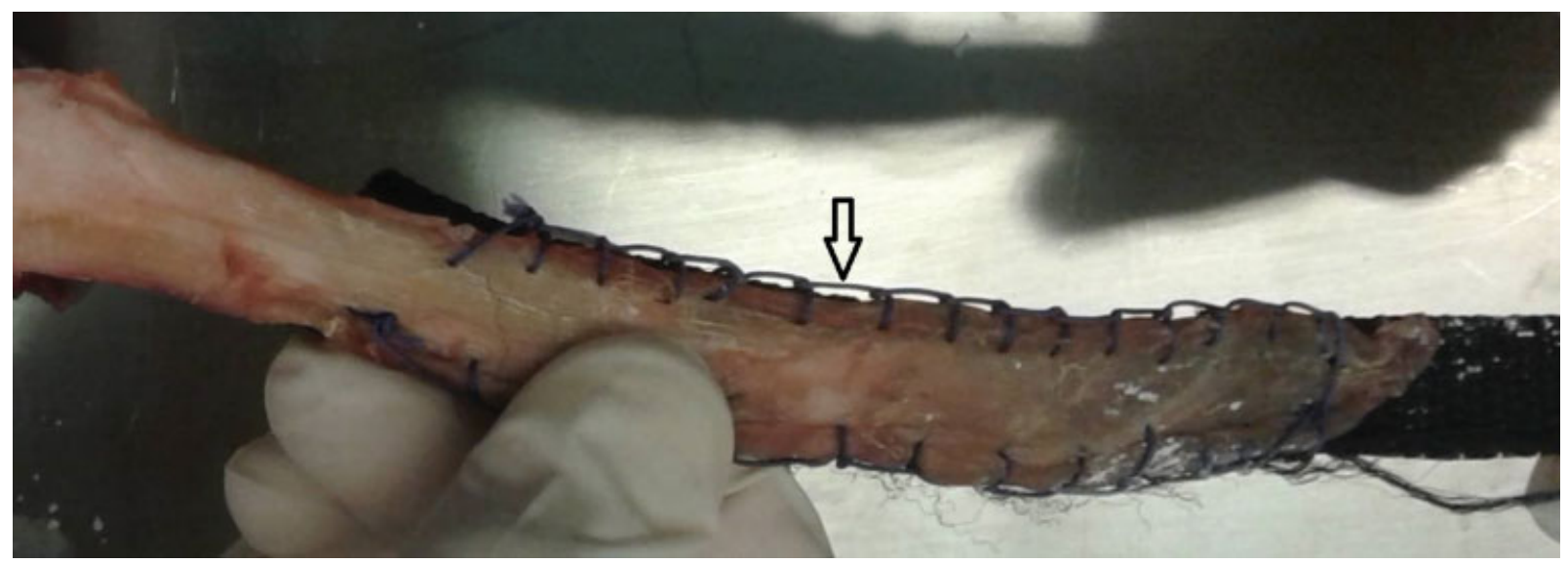

Fig. 1 Disección anatómica de tendón infraespinoso de cordero. Disección anatómica estandarizada, eliminando todo el tejido blando en relación a la diáfisis humeral, las fosas subescapular y supraespinosa de la escápula, con el objetivo de aislar el músculo infraespinoso y su tendón. El tendón fue suturado a una cinta de nailon polipropileno con una sutura tipo Krackow con MaxBraid \#2, con tal de poder manipular el músculo y tendón sin desgarrarlos. 


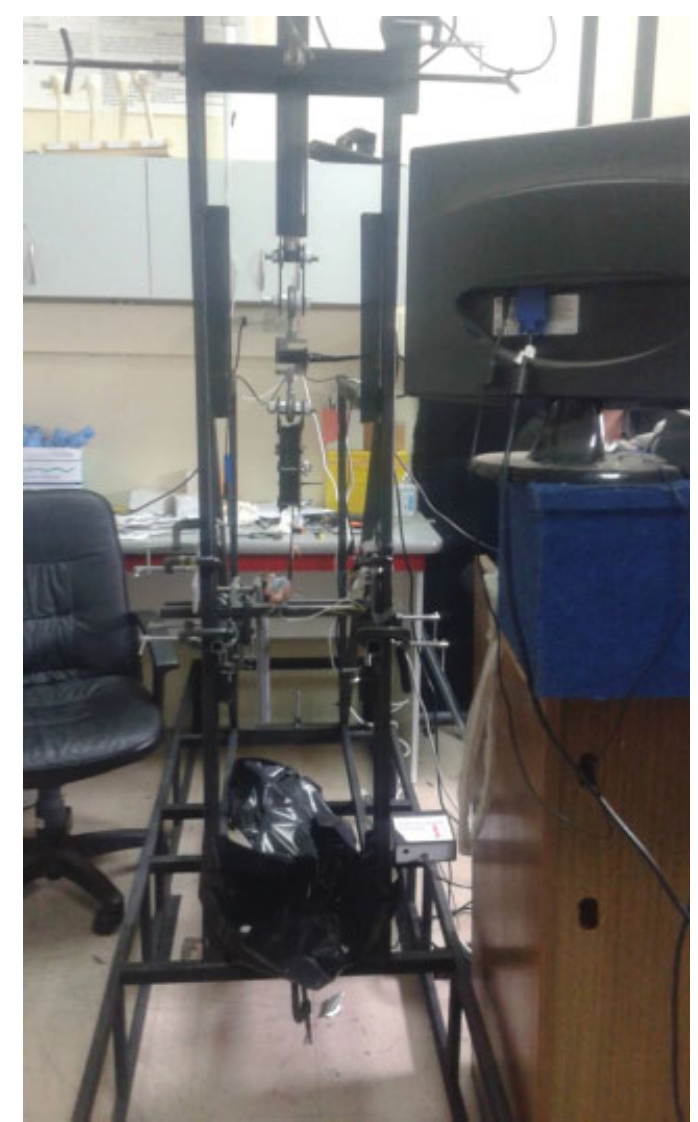

Fig. 2 Modelo de tensión cíclica. El modelo constó de tres partes fundamentales: un soporte modular de altura regulable; un soporte ajustable para guiar el sistema de suturas; y un actuador lineal, con una celda de carga intermedia. La diáfisis humeral fue fijada en una abrazadera metálica.

la aplicación de carga cíclica (cada 50 ciclos) y al final de la intervención (1.000 ciclos).

\section{Reparación de rotura de manguito rotador con suturas transóseas y sutura Mason-Allen modificada}

Para la reparación, se utilizó un sutura de polímero no absorbible trenzada de polietileno \#2 (MaxBrad), correspondiente al tamaño más comúnmente utilizado en la cirugía artroscópica de hombro. Los túneles transóseos fueron realizados con un dispositivo previamente diseñado por nuestro equipo y utilizado en modelos previos para la generación de túneles de arquitectura oblicua (-Figura 5).

Se realizaron dos reparaciones diferentes: 2 túneles TOCs de suturas MaxBraid \#2 ( $n=4)$ (-Figura 6a); y dos puntos MAMs con dos anclas Ti-Screw de titanio doble cargadas con MaxBraid \#2 $(n=4)$ (-Figura 6b).

Se realizó una pretensionado de la reparación con $10 \mathrm{~N}$ por 2 minutos. Luego, se programó la celda de carga a 1.000 ciclos, con una frecuencia $2 \mathrm{~Hz}$ y una carga de $30 \mathrm{~N}$. Estos parámetros se definieron con base en los utilizados en estudios anteriores similares, y refleja el período de rehabilitación inicial (dos semanas) en el postoperatorio a través de ejercicios pasivos con movimientos pendulares. ${ }^{32,33}$ No se registraron fallas a nivel de la reparación ni a nivel de los tejidos o soporte en relación a las cargas utilizadas.

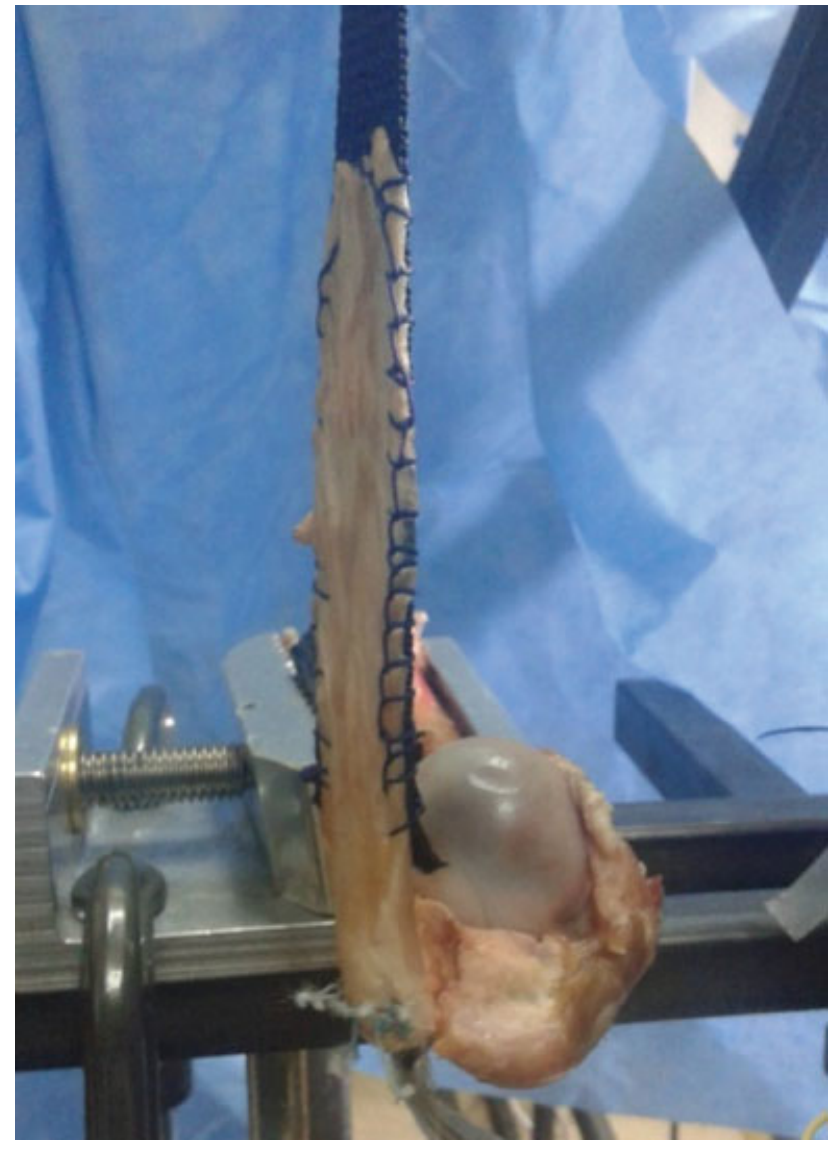

Fig. 3 Soporte modular. El soporte modular fue ajustado para asegurar el paralelismo del tendón con la vertical.

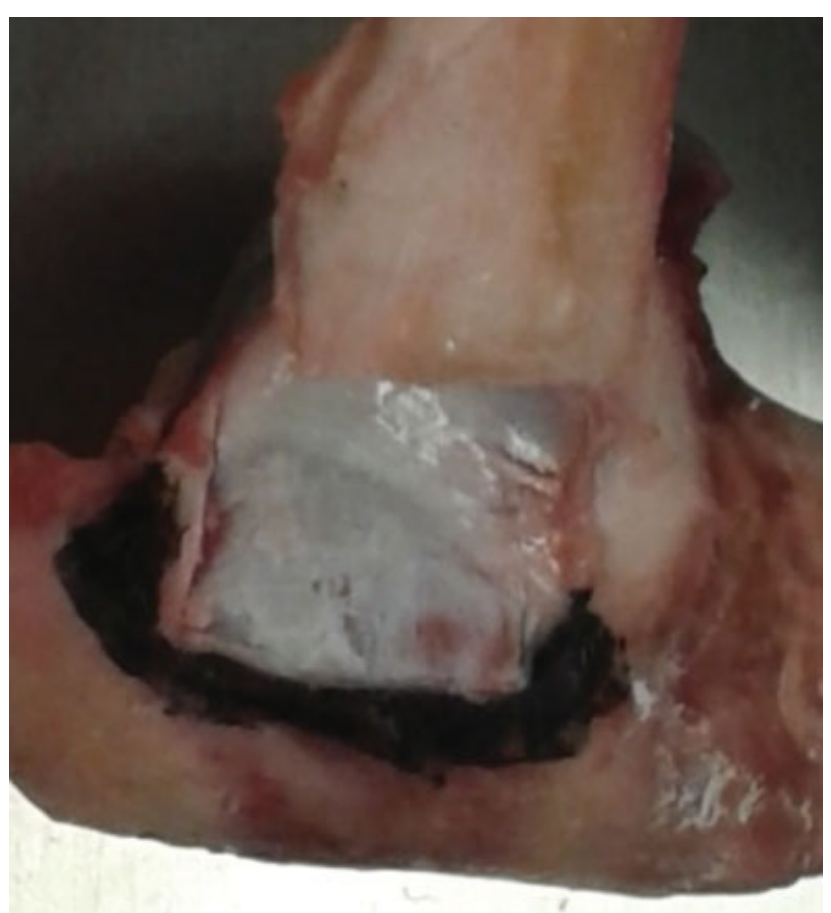

Fig. 4 Rotura del manguito rotador. Se realiza una rotura de espesor total y ancho completo con un bisturí $n^{\circ} 15$. La imagen muestra la huella del tendón infraespinoso. 


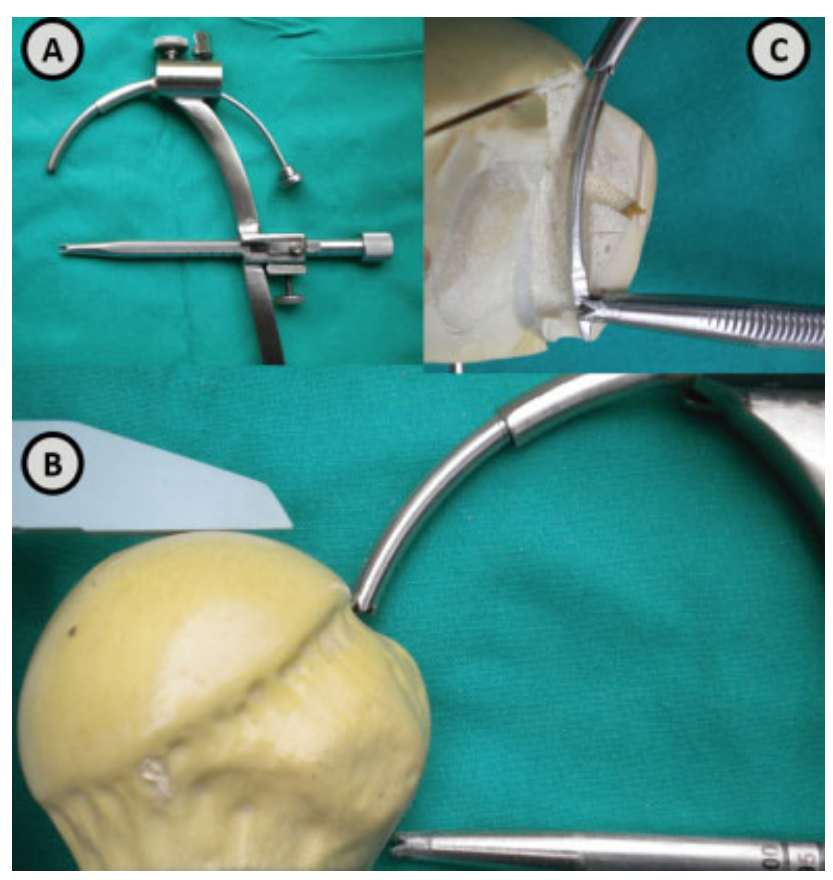

Fig. 5 Dispositivo para diseño de túneles transóseos oblicuos. En el recuadro $A$, se observa el dispositivo de suturas transóseas utilizado. En el recuadro B, se observa el posicionamiento adecuado del instrumento en relación a la tuberosidad mayor. Finalmente, en el recuadro $\mathrm{C}$, se observa un corte de un modelo Sawbone (Pacific Research Laboratories, Vashon, WA, EEUU) que muestra la trayectoria oblicua del túnel.
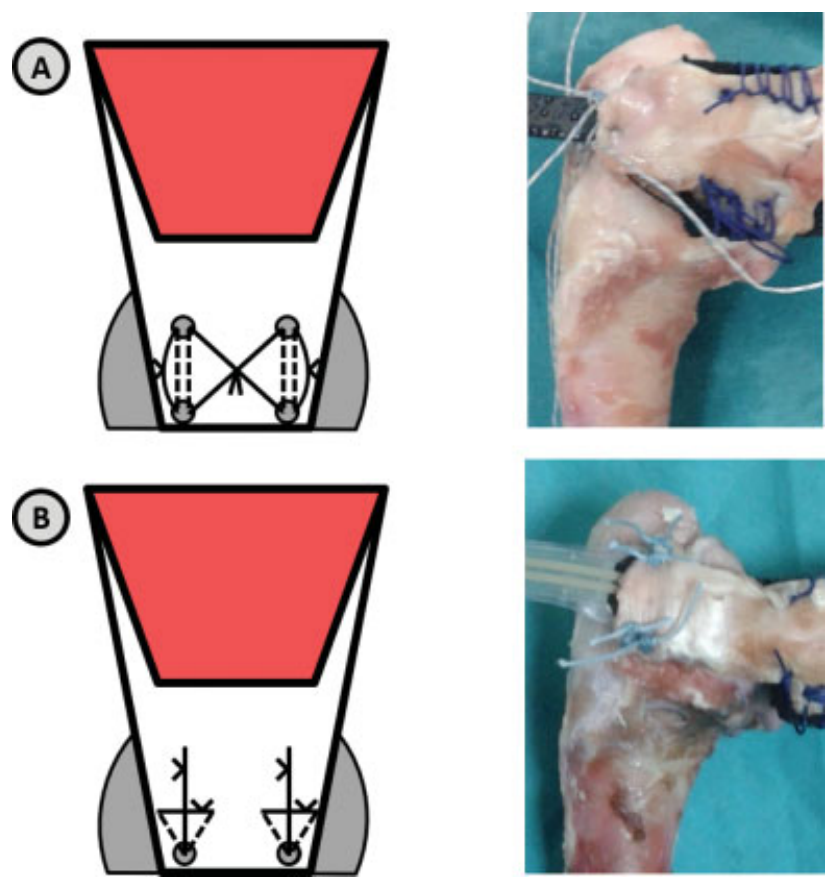

Fig. 6 Configuraciones de reparación. En el recuadro A, se observa la reparación transósea con nudos cruzados, y en el $\mathbf{B}$, la reparación con puntos Mason-Allen modificados.

\section{Análisis estadístico}

Los resultados fueron presentados como promedio \pm desviación estándar. Dada una distribución normal (comprobada por el test de Shapiro-Wilk), se utilizó la prueba estadística para variables paramétricas (test $t$ de Student). Todos los datos se analizaron utilizando el programa Stata (StataCorp., College Station, TX, EEUU), versión 14. La significancia estadística se consideró con valores de $p<0,05$.

\section{Resultados}

La presión en la interfase tendón-huella en respuesta a carga cíclica (medido con sensor de presión digital) en ambos modelos de reparación presentaró el mecanismo de autorreforzamiento durante los aumentos de tensión cíclica por parte del sistema (-Figura 7).

Se comparó el promedio de curva de presión de contacto en la interfase tendón-huella tras el ciclado y el promedio de presión de contacto residual final (tiempo $=1.000$ ciclos) en la interfase tendón-huella. El promedio de curva de presión de contacto en las piezas que fueron reparadas con suturas TOCs fue de $86,01 \pm 8,43 \%$, mientras que con MAM fue de $73,28 \pm 12,01 \%(p<0,0004)$. El promedio del porcentaje residual al final del ciclado fue de $71,57 \%$ para suturas TOCs y de $51,19 \%$ para MAM $(p<0,05)$ ( - Figura 8 ).

\section{Discusión}

El hallazgo principal de este estudio fue que la reparación TOC presenta mayor promedio de curva de presión de contacto y mayor porcentaje de presión de contacto residual final en la interfase tendón-huella que la reparación con sutura MAM luego de carga cíclica estandarizada. Esto podría estar relacionado con mejores tasas de cicatrización a nivel de la huella, y por ende, con mejores resultados clínicos.

Esto es comparable con estudios biomecánicos ${ }^{5,13}$ de la reparación en DF, que han demostrado un aumento de la resistencia a la falla por carga, áreas de contacto y presiones mejoradas, y una disminución de la formación de espacios (gaps) en la interfase tendón-huella, en comparación con la reparación en FU.

Caldow et al. ${ }^{9}$ demostraron inferioridad biomecánica de la técnica TO (área de contacto, presión de contacto, resistencia a la tracción y la rigidez) en comparación con MAM y DF. La presión de contacto para la reparación TO fue significativamente menor que la reparación MAM y la en DF. La reparación MAM demostró una resistencia a la tracción máxima significativamente mayor en comparación con la reparación TO; la reparación en DF presentó una resistencia a la tracción máxima significativamente mayor en comparación con la MAM y la TO. Sin embargo, estos resultados fueron hallados en un modelo de sutura TO simple, y no fue evaluada la reparación cruzada. En un estudio no publicado por nuestro grupo, las propiedades biomecánicas de la configuración cruzada en comparación con la configuración simple fueron superiores en área de contacto y presión a nivel de la huella.

Hinse et al. ${ }^{34}$ compararon TO con sutura, TO con cinta, y TO equivalente, encontrando que la carga a la falla no presentaba diferencias entre TO con cinta y TO 


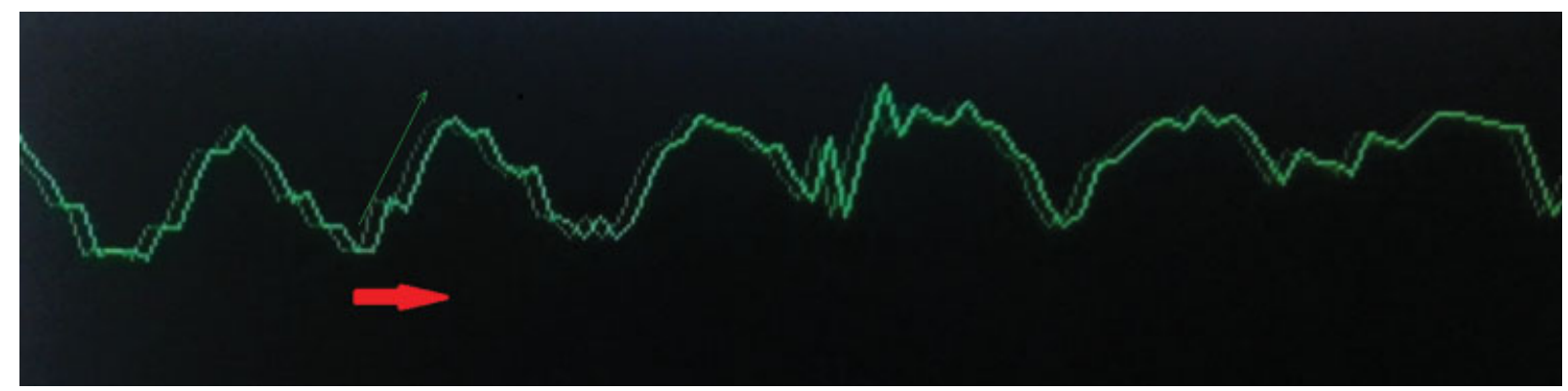

Fig. 7 Mecanismo de autorreforzamiento en reparación transósea. Ejemplo de medición de la presión ante cargas cíclicas. Esta demuestra el fenómeno de autorreforzamiento en reparación transósea. La flecha roja representa la tracción ejercida sobre el tendón. La flecha verde muestra como aumenta la presión a nivel de la huella en relación a la carga aplicada.

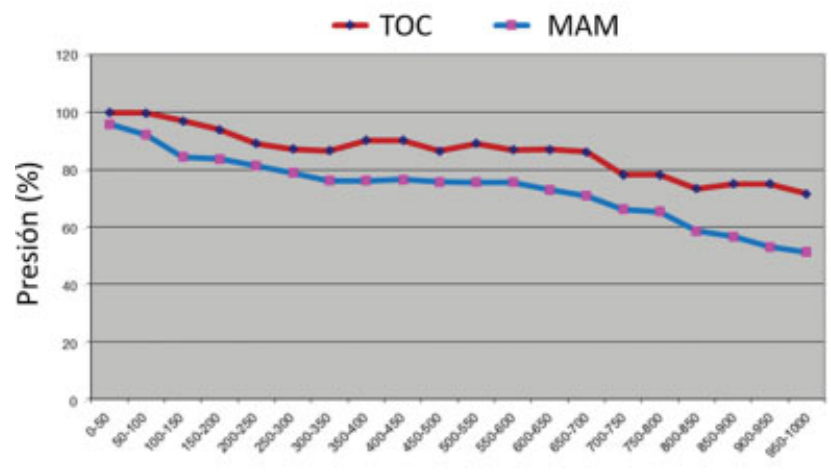

Ciclos de cargas

Fig. 8 Porcentaje de Presión a lo largo de carga cíclica. Esta figura muestra como disminuye la presión (expresada en porcentaje) a los largo de los ciclos de carga sobre ambas reparaciones. Abreviaturas: TOC, sutura transósea cruzada; MAM, Mason-Allen modificado.

equivalente, pero que TO con sutura tenía menor resistencia estadísticamente significativa en comparación con TO equivalente, por lo que el material utilizado podría ser un factor importante a considerar. Además, a pesar de no encontrar diferencias significativas, existe una tendencia en este estudio a una mayor pérdida de cobertura en la huella con las técnicas TOs puras. En este estudio, utilizamos MaxBraid \#2, una sutura de alta resistencia reconocida por presentar propiedades abrasivas menores en comparación con suturas similares; ${ }^{35}$ sin embargo, no existen trabajos comparativos con cinta en este tipo de modelo. Podemos inferir que la utilización de cintas del mismo material entregarían mayor resistencia a la falla. Sin embargo, bajo las cargas de una rehabilitación inicial, en este modelo, no registramos fallas del material.

Park et al. ${ }^{12}$ compararon TO simple, FU con puntos simples, y FU con puntos colchoneros, demostrando que la técnica de reparación del manguito rotador del túnel TO crea un contacto significativamente mayor y una mayor distribución general de la presión sobre la huella definida en comparación con las otras técnicas. Sin embargo, no compararon TOC ni MAM, que son las técnicas evaluadas en este estudio. Tuoheti et al ${ }^{36}$ compararon TO simple, con FU y DF, y encontraron superioridad de la DF en comparación con la TO; sin embargo, es una técnica TO simple y la DF con puntos colchoneros, que presenta debilidades similares al las del estudio de Park et al. ${ }^{12}$

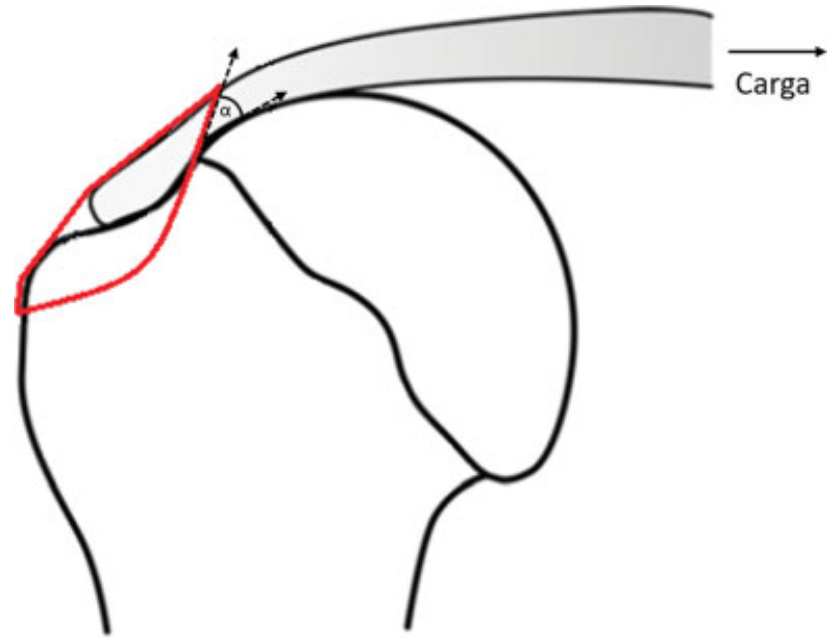

Fig. 9 Esquema de autorreforzamiento en reparación transósea. Esquema que ilustra un acuñamiento del ángulo entre el material de sutura y el hueso a medida que el tendón se tensiona progresivamente, y un cambio de geometría rectangular a trapezoidal de las suturas en el plano coronal a medida que aumenta la carga de tracción.

Sin embargo, estos estudio sólo evalúan propiedades biomecánicas en relación a magnitud y distribución de la presión, además de carga a la falla, y, al parecer, la técnica TO tiene beneficios para la cicatrización a nivel del aporte de células mesenquimales y una mejor vascularización del tendón. ${ }^{24-26}$ Urita et al. $^{37}$ demostraron que la vascularización en pacientes estudiados con ultrasonido es superior en aquellos con reparación artroscópica TO en comparación con TO equivalente. Esto podría reflejar otro beneficio de la técnica TO que podría mejorar la cicatrización.

El autorreforzamiento es un mecanismo descrito por Burkhart et al. ${ }^{27}$ el año 2009, por el cual el aumento de la tensión aplicado al constructo genera una resistencia creciente a la falla estructural, al generar un aumento progresivo de las fuerzas de compresión en la huella del tendón. Las fuerzas de compresión creadas en la huella aumentan la resistencia a la fricción entre el tendón y el hueso, reduciendo así la formación de espacios entre las dos superficies. ${ }^{27}$ Este fenómeno fue observado en ambas técnicas de reparación. Probablemente, se produce un acuñamiento del ángulo entre el material de sutura y el hueso a medida que el tendón se tensiona progresivamente, y un cambio de geometría rectangular a 
trapezoidal de las suturas en el plano coronal a medida que aumenta la carga de tracción. ${ }^{27}$ Esto provoca una deformación elástica del tendón, creando una fuerza de compresión perpendicular a la superficie del hueso, que aumenta a medida que aumenta la carga de tracción (- Figura 9). ${ }^{27}$

Finalmente, hay que considerar que además de las ventajas biomecánicas y biológicas que puede ofrecer la técnica TO, puede llegar a ser una técnica con mayor costoefectividad, por la reutilización del dispositivo y el bajo costo de las suturas de alta resistencia en comparación con otros tipos de constructos. Sin embargo, estas aseveraciones no fueron evaluadas en esta investigación.

Dentro de las limitaciones, este estudio evaluó sólo los aspectos biomecánicos en un modelo animal, por lo que los hallazgos podrían ser diferentes en un humano y en condiciones biológicas (considerar células mesenquimáticas y la irrigación). Hombros cadavéricos humanos representarían mejor a la población clínica. Por otro lado, el uso de este modelo estandariza nuestros resultados, porque cada muestra tiene seis meses de edad, y, por lo tanto, es fácilmente comparable a las demás. Esto también es cierto para la densidad mineral ósea, que no se calculó para nuestras muestras, pero habría sido muy similar con las muestras teniendo la misma edad.

Concluimos que la reparación TOC presenta propiedades biomecánicas superiores en la interfase tendón-huella a la reparación con sutura MAM luego de carga cíclica estandarizada. Esto podría tener repercusiones clínicas importantes en las tasas de cicatrización y resultados funcionales de los pacientes, pero hay que evaluar la aplicabilidad de este dispositivo en el ambiente quirúrgico y la posibilidad de su utilización a través de una técnica artroscópica.

\section{Conflicto de Intereses}

Los autores declaran que no hay conflicto de intereses.

Agradecimientos

A nuestra familia, por el constante apoyo en nuestra labor de investigación.

\section{Referencias}

1 Jancuska J, Matthews J, Miller T, Kluczynski MA, Bisson LJ. A Systematic Summary of Systematic Reviews on the Topic of the Rotator Cuff. Orthop J Sports Med 2018;6(09):2325967118797891. Doi: $10.1177 / 2325967118797891$

2 Collin P, Colmar M, Thomazeau H, et al. Clinical and MRI Outcomes 10 Years After Repair of Massive Posterosuperior Rotator Cuff Tears. J Bone Joint Surg Am 2018;100(21):1854-1863. Doi: 10.2106/JBJS.17.01190

3 Collin P, Thomazeau H, Walch G, et al. Clinical and structural outcome twenty years after repair of isolated supraspinatus tendon tears. J Shoulder Elbow Surg 2019;28(01):196-202. Doi: 10.1016/j.jse.2018.07.023

4 Piper CC, Hughes AJ, Ma Y, Wang H, Neviaser AS. Operative versus nonoperative treatment for the management of full-thickness rotator cuff tears: a systematic review and meta-analysis. J Shoulder Elbow Surg 2018;27(03):572-576. Doi: 10.1016/j. jse.2017.09.032

5 Rossi LA, Rodeo SA, Chahla J, Ranalletta M. Current Concepts in Rotator Cuff Repair Techniques: Biomechanical, Functional, and
Structural Outcomes. Orthop J Sports Med 2019;7(09): 2325967119868674. Doi: $10.1177 / 2325967119868674$

6 Chona DV, Lakomkin N, Lott A, et al. The timing of retears after arthroscopic rotator cuff repair. J Shoulder Elbow Surg 2017;26 (11):2054-2059. Doi: 10.1016/j.jse.2017.07.015

7 Haque A, Pal Singh H. Does structural integrity following rotator cuff repair affect functional outcomes and pain scores? A metaanalysis. Shoulder Elbow 2018;10(03):163-169. Doi: 10.1177/ 1758573217731548

8 Galatz LM, Ball CM, Teefey SA, Middleton WD, Yamaguchi K. The outcome and repair integrity of completely arthroscopically repaired large and massive rotator cuff tears. J Bone Joint Surg Am 2004;86(02):219-224. Doi: 10.2106/00004623-20040200000002

9 Caldow J, Richardson M, Balakrishnan S, Sobol T, Lee PV, Ackland DC. A cruciate suture technique for rotator cuff repair. Knee Surg Sports Traumatol Arthrosc 2015;23(02):619-626. Doi: 10.1007/ s00167-014-3474-7

10 Desmoineaux P. Failed rotator cuff repair. Orthop Traumatol Surg Res 2019;105(1S):S63-S73. Doi: 10.1016/j.otsr.2018.06.012

11 Cicak N, Klobucar H, Bicanic G, Trsek D. Arthroscopic transosseous suture anchor technique for rotator cuff repairs. Arthroscopy 2006;22(05):565.e1-565.e6. Doi: 10.1016/j.arthro.2005.07.029

12 Park MC, Cadet ER, Levine WN, Bigliani LU, Ahmad CS. Tendon-tobone pressure distributions at a repaired rotator cuff footprint using transosseous suture and suture anchor fixation techniques. Am J Sports Med 2005;33(08):1154-1159. Doi: 10.1177/ 0363546504273053

13 Hohmann E, König A, Kat CJ, Glatt V, Tetsworth K, Keough N. Single- versus double-row repair for full-thickness rotator cuff tears using suture anchors. A systematic review and metaanalysis of basic biomechanical studies. Eur J Orthop Surg Traumatol 2018;28(05):859-868. Doi: 10.1007/s00590-0172114-6

14 Apreleva M, Ozbaydar M, Fitzgibbons PG, Warner JJ. Rotator cuff tears: the effect of the reconstruction method on threedimensional repair site area. Arthroscopy 2002;18(05): 519-526. Doi: 10.1053/jars.2002.32930

15 Ma R, Chow R, Choi L, Diduch D. Arthroscopic rotator cuff repair: suture anchor properties, modes of failure and technical considerations. Expert Rev Med Devices 2011;8(03):377-387. Doi: $10.1586 /$ erd.11.4

16 Kowalsky MS, Dellenbaugh SG, Erlichman DB, Gardner TR, Levine $\mathrm{WN}$, Ahmad CS. Evaluation of suture abrasion against rotator cuff tendon and proximal humerus bone. Arthroscopy 2008;24(03): 329-334. Doi: 10.1016/j.arthro.2007.09.011

17 Ntalos D, Huber G, Sellenschloh K, et al. All-suture anchor pullout results in decreased bone damage and depends on cortical thickness. Knee Surg Sports Traumatol Arthrosc 2020;•••;. Doi: 10.1007/s00167-020-06004-6

18 Godry H, Jettkant B, Seybold D, Venjakob AJ, Bockmann B. Pullout strength and failure mode of industrially manufactured and selfmade all-suture anchors: a biomechanical analysis. J Shoulder Elbow Surg 2020;29(07):1479-1483

19 Imam MA, Abdelkafy A. Outcomes following arthroscopic transosseous equivalent suture bridge double row rotator cuff repair: a prospective study and short-term results. SICOT J 2016; 2:7. Doi: $10.1051 /$ sicotj/2015041

20 Flanagin BA, Garofalo R, Lo EY, et al. Midterm clinical outcomes following arthroscopic transosseous rotator cuff repair. Int J Shoulder Surg 2016;10(01):3-9. Doi: 10.4103/0973-6042.174511

21 Garofalo R, Castagna A, Borroni M, Krishnan SG. Arthroscopic transosseous (anchorless) rotator cuff repair. Knee Surg Sports Traumatol Arthrosc 2012;20(06):1031-1035. Doi: 10.1007/ s00167-011-1725-4

22 Benson EC, MacDermid JC, Drosdowech DS, Athwal GS. The incidence of early metallic suture anchor pullout after 
arthroscopic rotator cuff repair. Arthroscopy 2010;26(03): 310-315. Doi: 10.1016/j.arthro.2009.08.015

23 Chillemi C, Mantovani M. Arthroscopic trans-osseous rotator cuff repair. Muscles Ligaments Tendons J 2017;7(01):19-25. Doi: 10.11138/mltj/2017.7.1.019

24 Tauber M, Koller H, Resch H. Transosseous arthroscopic repair of partial articular-surface supraspinatus tendon tears. Knee Surg Sports Traumatol Arthrosc 2008;16(06):608-613. Doi: 10.1007/ s00167-008-0532-z

25 Campbell TM, Lapner P, Dilworth FJ, et al. Tendon contains more stem cells than bone at the rotator cuff repair site. J Shoulder Elbow Surg 2019;28(09):1779-1787. Doi: 10.1016/j.jse.2019.02.008

26 Kida Y, Morihara T, Matsuda K, et al. Bone marrow-derived cells from the footprint infiltrate into the repaired rotator cuff. J Shoulder Elbow Surg 2013;22(02):197-205. Doi: 10.1016/j. jse.2012.02.007

27 Burkhart SS, Adams CR, Burkhart SS, Schoolfield JD. A biomechanical comparison of 2 techniques of footprint reconstruction for rotator cuff repair: the SwiveLock-FiberChain construct versus standard double-row repair. Arthroscopy 2009;25 (03):274-281. Doi: 10.1016/j.arthro.2008.09.024

28 Scheibel MT, Habermeyer P. A modified Mason-Allen technique for rotator cuff repair using suture anchors. Arthroscopy 2003;19 (03):330-333. Doi: 10.1053/jars.2003.50079

29 Lichtenberg S, Siebold R, Habermeyer P. Arthroscopic supraspinatus tendon repair using suture anchors and a modified Mason-Allen technique: an intra-articular approach. Arthroscopy 2004;20(09): 1007-1011. Doi: 10.1016/j.arthro.2004.07.004

30 Lichtenberg S, Liem D, Magosch P, Habermeyer P. Influence of tendon healing after arthroscopic rotator cuff repair on clinical outcome using single-row Mason-Allen suture technique: a prospective, MRI controlled study. Knee Surg Sports Traumatol
Arthrosc 2006;14(11):1200-1206. Doi: 10.1007/s00167-0060132-8

31 Andres BM, Lam PH, Murrell GA. Tension, abduction, and surgical technique affect footprint compression after rotator cuff repair in an ovine model. J Shoulder Elbow Surg 2010;19(07):1018-1027. Doi: $10.1016 /$ j.jse.2010.04.005

32 Mahar AT, Moezzi DM, Serra-Hsu F, Pedowitz RA. Comparison and performance characteristics of 3 different knots when tied with 2 suture materials used for shoulder arthroscopy. Arthroscopy 2006;22(06):614.e1-614.e2. Doi: 10.1016/j.arthro.2006.02.005

33 Wüst DM, Meyer DC, Favre P, Gerber C. Mechanical and handling properties of braided polyblend polyethylene sutures in comparison to braided polyester and monofilament polydioxanone sutures. Arthroscopy 2006;22(11):1146-1153. Doi: 10.1016/j.arthro.2006.06.013

34 Hinse S, Ménard J, Rouleau DM, Canet F, Beauchamp M Biomechanical study comparing 3 fixation methods for rotator cuff massive tear: Transosseous No. 2 suture, transosseous braided tape, and double-row. J Orthop Sci 2016;21(06): 732-738. Doi: 10.1016/j.jos.2016.07.001

35 Williams JF, Patel SS, Baker DK, Schwertz JM, McGwin G, Ponce BA. Abrasiveness of high-strength sutures used in rotator cuff surgery: are they all the same? J Shoulder Elbow Surg 2016;25 (01):142-148. Doi: 10.1016/j.jse.2015.07.018

36 Tuoheti Y, Itoi E, Yamamoto $\mathrm{N}$, et al. Contact area, contact pressure, and pressure patterns of the tendon-bone interface after rotator cuff repair. Am J Sports Med 2005;33(12): 1869-1874. Doi: 10.1177/0363546505278256

37 Urita A, Funakoshi T, Horie T, Nishida M, Iwasaki N. Difference in vascular patterns between transosseous-equivalent and transosseous rotator cuff repair. J Shoulder Elbow Surg 2017;26 (01):149-156. Doi: 10.1016/j.jse.2016.06.010 